\title{
Phacoemulsification versus peripheral iridotomy in the management of chronic primary angle closure: long-term follow-up
}

\author{
Arnaldo Dias-Santos · Joana Ferreira • \\ Luís Abegão Pinto • Isabel Domingues • \\ José Pedro Silva · João Paulo Cunha $\cdot$ Maria Reina
}

Received: 23 November 2013/ Accepted: 20 February 2014/Published online: 12 April 2014

(C) Springer Science+Business Media Dordrecht 2014

\begin{abstract}
Primary angle closure occurs as a result of crowded anterior segment anatomy, causing appositional contact between the peripheral iris and trabecular meshwork, thereby obstructing aqueous outflow. Several studies highlight the role of the crystalline lens in its pathogenesis. The objective of this work is to compare the long-term efficacy of phacoemulsification versus laser peripheral iridotomy (LPI) in the management of chronic primary angle closure (CPAC). Prospective case-control study with 30 eyes of 30 patients randomly divided in two groups: 15 eyes in the LPI group and 15 eyes in the IOL group. Patients in the LPI group underwent LPI using argon and Nd:YAG laser. Patients in the IOL group underwent
\end{abstract}

This work was presented as a poster presentation at the American Academy of Ophthalmology Annual Meeting, November, 2013.

A. Dias-Santos $(\varangle) \cdot$ J. Ferreira $\cdot$ L. Abegão Pinto ·

I. Domingues - J. P. Cunha · M. Reina

Department of Ophthalmology, Central Lisbon Hospital

Center, Lisbon, Portugal

e-mail: arnaldomiguelsantos@gmail.com

A. Dias-Santos

Serviço de Oftalmologia, Hospital de Santo António dos Capuchos, Alameda de Santo António dos Capuchos, 1169-050 Lisboa, Portugal

J. P. Silva

Department of Ophthalmology, HPP Lusíadas Hospital, Lisbon, Portugal phacoemulsification with posterior chamber intraocular lens (IOL) implantation. Examinations before and after the procedure included gonioscopy, Goldmann applanation tonometry, and anterior chamber evaluation using the Pentacam rotating Scheimpflug camera. The mean follow-up time was $31.13 \pm 4.97$ months. There was a statistically significant reduction in the intraocular pressure (IOP) and number of anti-glaucoma medications $(p<0.01)$ only in the IOL group. Anterior chamber depth, angle, and volume were all higher in the IOL group $(p<0.01)$ at the end of the follow-up period. Phacoemulsification with posterior chamber IOL implantation results in a higher anterior chamber depth, angle, and volume, when compared to LPI. Consequently, phacoemulsification has greater efficacy in lowering IOP and preventing its long-term increase in patients with CPAC and cataract.

Keywords Cataract - Chronic primary angle closure $\cdot$ Laser peripheral iridotomy ·

Phacoemulsification

\section{Introduction}

Primary angle closure (PAC) occurs as a result of crowded anterior segment anatomy, causing appositional contact between the peripheral iris and trabecular meshwork, thereby obstructing aqueous outflow [1]. By the time this blockage causes pressure-induced optic neuropathy, the condition is called primary angle closure 
glaucoma (PACG). PACG accounts for half of all glaucoma blindness worldwide [2]. PACG or PAC and cataract often coexist and the crystalline lens plays a pivotal role in their pathogenesis. The cardinal anatomic characteristic in eyes with angle closure is a thicker, more anteriorly positioned crystalline lens in comparison to unaffected eyes. Thickening and forward movement of the lens occur with aging, which explains why PACG is more commonly found in older patients [3].

Laser peripheral iridotomy (LPI) has been used widely for treating acute or chronic angle closure by relieving pupillary block, by far the most common cause of PAC. However, despite being very effective in preventing symptomatic acute pressure rises in PAC patients who have had one episode in their fellow eye $[4,5]$, it is not completely effective in preventing longterm asymptomatic rises in intraocular pressure (IOP) [5]. Angle width increases in most eyes after LPI, but between 20 and $25 \%$ of eyes, show no change [6, 7]. Several studies have demonstrated the efficacy of cataract surgery in chronic angle closure glaucoma [8, 9], as well as in controlling IOP after an episode of acute angle closure glaucoma $[10,11]$. The objective of this study is to compare the long-term results of phacoemulsification with posterior chamber intraocular lens (IOL) implantation versus LPI in the management of chronic primary angle closure with cataract, in terms of clinical efficacy and anterior chamber parameters.

\section{Methods}

This study was conducted at Central Lisbon Hospital Center, a university-based tertiary center. Patients were prospectively recruited between January and June 2010. Written, informed consent was obtained from all subjects and this investigation adhered to the tenets of the Declaration of Helsinki. Ethics Committee approval was obtained. Patients with cataract and PAC or controlled PACG, without history of intraocular surgery or any other ocular disease were included in this study.

Diagnosis of PAC was made when gonioscopy revealed two or more quadrants of irido-trabecular contact with gaze in the primary position and another abnormality: either IOP $>21 \mathrm{mmHg}$ or peripheral anterior synechiae extending across the scleral spur, without iritis or any other signs of ocular pathology other than cataract [12]. Additionally, the presence of glaucomatous optic disk excavation, reproducible visual field abnormality or both leaded to the diagnosis of chronic PACG.

Thirty eyes of 30 patients included in the study were randomly divided in two groups: 15 eyes underwent LPI-LPI group — and 15 eyes underwent phacoemulsification with posterior chamber IOL implantationIOL group. Patient randomization was performed using a computer-based randomization program (www.randomization.com-accessed on 15th January 2010).

Phacoemulsification with IOL implantation was performed by two surgeons (JPC and JPS). Preoperative intravenous manitol $20 \%$ at $1-2 \mathrm{~g} / \mathrm{kg}$ was given $2 \mathrm{~h}$ before surgery for all patients. Under topical anesthesia, a $2.75 \mathrm{~mm}$ clear cornea incision was made. After continuous curvilinear capsulorhexis, standard phacoemulsification was performed using the "phacochop" technique. A posterior chamber foldable IOL with a $6.0 \mathrm{~mm}$ acrylic optic (Acrysof IQ, Alcon, Fort Worth, TX) was implanted in the capsular bag. At the end of the surgery, intracameral cefuroxime was injected. Postoperative treatment included topical steroids and antibiotics, tapered over 1 month.

Laser peripheral iridotomy was performed under topical anesthesia, using combined argon and Nd:YAG laser. It was sited at the superior nasal or superior temporal quadrant and the size of the opening was $\geq 200 \mu \mathrm{m}$. One drop of apraclonidine $1 \%$ was administered $1 \mathrm{~h}$ before the procedure. Postoperative treatment included 1 drop of apraclonidine $1 \%$ immediately after the procedure and topical steroids for 1 week. Supplementary laser iridotomy was performed when needed (small or closed iridotomy).

Visual acuity (VA), number of anti-glaucoma medications, gonioscopy, and IOP (Goldmann applanation tonometry was used, with three readings taken and the mean value recorded) were assessed in both groups. Morphologic examination of the anterior chamber using the Pentacam Scheimpflug camera (Pentacam HR, Oculus, Germany) was also performed and the following parameters were analyzed: anterior chamber depth (ACD), anterior chamber angle (ACA), and anterior chamber volume (ACV).

The data were statistically analyzed using SPSS for Windows, version 20.0; IBM/SPSS, Chicago, IL. Student's $t$ test was performed and a $p$ value of less than 0.01 was deemed statistically significant. All the results were expressed as mean \pm standard deviation, with ranges between brackets. 
Table 1 Preoperative intraocular pressure (IOP), number of anti-glaucoma drugs, best corrected visual acuity (BCVA), anterior chamber depth (ACD), anterior chamber volume (ACV), and anterior chamber angle (ACA)

\begin{tabular}{lccc}
\hline & LPI group & IOL group & $p$ value \\
\hline IOP $(\mathrm{mm} \mathrm{Hg})$ & $15.07 \pm 3.26(10-22)$ & $19.93 \pm 8.30(10-46)$ & 0.022 \\
No. drugs & $0.6 \pm 1.12(0-3)$ & $1.67 \pm 1.18(0-3)$ & $0.008 *$ \\
BCVA & $0.88 \pm 0.18(0.5-0.9)$ & $0.64 \pm 0.34(0.2-0.9)$ & 0.010 \\
ACD $(\mathrm{mm})$ & $2.02 \pm 0.29(1.52-2.42)$ & $1.82 \pm 0.33(1.07-2.23)$ & 0.045 \\
ACV $\left(\mathrm{mm}^{3}\right)$ & $88.27 \pm 21.72(47-112)$ & $77.60 \pm 17.96(39-100)$ & 0.077 \\
ACA $($ degrees $)$ & $22.40 \pm 4.39(17.1-30.1)$ & $24.85 \pm 6.42(9.0-33.2)$ & 0.116 \\
\hline
\end{tabular}

* Statistically significant difference between both groups

Table 2 Postoperative intraocular pressure (IOP), number of anti-glaucoma drugs, best corrected visual acuity (BCVA), anterior chamber depth (ACD), anterior chamber volume (ACV), and anterior chamber angle (ACA)

\begin{tabular}{lccr}
\hline & LPI group & IOL group & $p$ value \\
\hline IOP $(\mathrm{mm} \mathrm{Hg})$ & $14.87 \pm 2.19(10-18)$ & $14.53 \pm 1.51(11-17)$ & 0.316 \\
No. drugs & $0.40 \pm 0.83(0-3)$ & $0.93 \pm 0.59(0-2)$ & 0.026 \\
BCVA & $0.75 \pm 0.31(0.1-0.9)$ & $0.87 \pm 0.21(0.2-1.0)$ & 0.280 \\
ACD $(\mathrm{mm})$ & $1.99 \pm 0.27(1.57-2.45)$ & $3.87 \pm 0.48(3.36-5.04)$ & $<0.001^{*}$ \\
ACV $\left(\mathrm{mm}^{3}\right)$ & $102.47 \pm 19.15(68-135)$ & $159.67 \pm 23.01(125-196)$ & $<0.001^{*}$ \\
ACA $($ degrees $)$ & $23.05 \pm 3.06(19.0-29.9)$ & $43.98 \pm 8.71(30.9-56.2)$ & $<0.001^{*}$ \\
\hline
\end{tabular}

* Statistically significant difference between both groups

\section{Results}

Thirty patients were enrolled in the study: 15 in the LPI group, 3 men and 12 women; and 15 in the IOL group, 4 men and 11 women. The mean age in the LPI group was $65.10 \pm 9.49$ (44-76) years and the mean age in the IOL group was $69.5 \pm 11.34(52-86)$ years $(p=0.180)$. Preoperative clinical and ocular data are shown in Table 1 . There was a statistically significant difference only in the number of preoperative drugs, which was higher in the IOL group.

The mean follow-up time was $31.13 \pm 4.97$ months.

The postoperative clinical and ocular data for both groups are shown in Table 2. There were statistically significant differences for all the anterior chamber parameters determined with the Pentacam, which were higher in the IOL group, but not for IOP, number of anti-glaucoma drugs or BCVA. $93.3 \%$ (14 eyes in 15) of the eyes in the LPI group maintained two or more quadrants of iridocorneal contact on gonioscopy, while none of the eyes in the IOL group presented two or more quadrants of iridocorneal contact after the follow-up period.
Tables 3 and 4 compare the pre and postoperative data for LPI and IOL groups, respectively. There were no statistically significant differences between pre and postoperative IOP, number of anti-glaucoma medications, BCVA or anterior chamber parameters in the LPI group. In the IOL group, the IOP and number of anti-glaucoma medications were significantly lower and all the anterior chamber parameters were higher after the follow-up period $(p<0.01)$.

There were no complications during or after any of the surgical procedures, and there were no cases of acute angle closure in any of the eyes enrolled in the study during the whole follow-up.

\section{Discussion}

The results of this study suggest that phacoemulsification with posterior chamber IOL implantation significantly reduced IOP and the number of antiglaucoma medications after a long-term follow-up. On the other hand, LPI did not significantly decrease the IOP or the number of anti-glaucoma medications. 
Table 3 Pre and postoperative intraocular pressure (IOP), number of anti-glaucoma drugs, best corrected visual acuity (BCVA), anterior chamber depth (ACD), anterior chamber volume (ACV), and anterior chamber angle (ACA) for patients in the LPI group

\begin{tabular}{lccc}
\hline & Before LPI & After LPI & $p$ value \\
\hline IOP (mm Hg) & $15.07 \pm 3.26(10-22)$ & $14.87 \pm 2.19(10-18)$ & 0.423 \\
No. drugs & $0.6 \pm 1.12(0-3)$ & $0.40 \pm 0.83(0-3)$ & 0.291 \\
BCVA & $0.88 \pm 0.18(0.5-0.9)$ & $0.75 \pm 0.31(0.1-0.9)$ & 0.064 \\
ACD $(\mathrm{mm})$ & $2.02 \pm 0.29(1.52-2.42)$ & $1.99 \pm 0.27(1.57-2.45)$ & 0.416 \\
ACV $\left(\mathrm{mm}^{3}\right)$ & $88.27 \pm 21.72(47-112)$ & $102.47 \pm 19.15(68-135)$ & 0.034 \\
ACA (degrees) & $22.40 \pm 4.39(17.1-30.1)$ & $23.05 \pm 3.06(19.0-29.9)$ & 0.322 \\
\hline
\end{tabular}

Table 4 Pre and postoperative intraocular pressure (IOP), number of anti-glaucoma drugs, best corrected visual acuity (BCVA), anterior chamber depth (ACD), anterior chamber volume (ACV), and anterior chamber angle (ACA) for patients in the IOL group

\begin{tabular}{lccr}
\hline & Before IOL & After IOL & $p$ value \\
\hline IOP (mm Hg) & $19.93 \pm 8.30(10-46)$ & $14.53 \pm 1.51(11-17)$ & $0.009 *$ \\
No. drugs & $1.67 \pm 1.18(0-3)$ & $0.93 \pm 0.59(0-2)$ & $0.003 *$ \\
BCVA & $0.64 \pm 0.34(0.2-0.9)$ & $0.87 \pm 0.21(0.2-1.0)$ & 0.015 \\
ACD (mm) & $1.82 \pm 0.33(1.07-2.23)$ & $3.87 \pm 0.48(3.36-5.04)$ & $<0.001 *$ \\
ACV $\left(\mathrm{mm}^{3}\right)$ & $77.60 \pm 17.96(39-100)$ & $159.67 \pm 23.01(125-196)$ & $<0.001^{*}$ \\
ACA (degrees) & $24.85 \pm 6.42(9.0-33.2)$ & $43.98 \pm 8.71(30.9-56.2)$ & $<0.001^{*}$ \\
\hline
\end{tabular}

* Statistically significant difference

Analyzing the preoperative clinical data of both groups, one can see that the number of anti-glaucoma medications was significantly higher in the phacoemulsification group. The preoperative IOP values were also higher (although not statistically significant) in the IOL group. This was probably the result of the limited sample size and explains why we didn't obtain statistically significant differences in these variables between the two groups after the follow-up. However, comparing preoperative and postoperative values, there are statistically significant differences only in the IOL group. Regarding the anterior chamber parameters, there were significant increments in the ACD, volume, and angle in the IOL group, but not in the LPI group. This is probably related to the pathophysiological mechanisms that increase the aqueous drainage after cataract surgery in these patients. Removal of the lens can deepen the anterior chamber and relieve the crowding of the angle [13, 14]. The stretching effect of the IOL in the bag could also open spaces in the trabecular meshwork that can explain the long-term decrease in the IOP [15]. Moreover Shams et al. [16] identified a significant reduction in peripheral anterior synechiae following cataract extraction alone, even without goniosynechialysis. Another possible mechanism that has been proposed to explain the improved aqueous outflow facility is the increase in prostaglandin release [15, 17]. The cytokine modulation triggered by the effect of ultrasound in the trabecular meshwork could also motivate an increase in its permeability [18]. In fact, the narrower the anterior chamber, the greater increase in the anterior chamber parameters and the higher effect in IOP after cataract surgery [19-21].

Other studies had already demonstrated the improvement in anterior chamber parameters after cataract surgery [22-26] but this, to our knowledge, has the longest follow-up, demonstrating the stability of these changes, as well as their effect in IOP control.

Laser peripheral iridotomy, which acts by relieving pupillary block, has been advocated as the initial treatment in eyes with PACG because of its noninvasive nature and safety [27]. However, despite its efficacy in preventing acute angle closure attacks, its effect in long-term control of IOP in eyes with chronic primary angle closure is more debatable [5]. The probable explanation for this fact is that a considerable number of patients have a mixed mechanism for angle 
closure [28, 29]. On the other hand, cataract surgery relieves angle closure by attenuating various causative factors like pupillary block, plateau iris, and lensassociated [13, 30]. Hata et al. [31] compared the efficacy of LPI versus phacoemulsification for the management of chronic angle closure, after 6 months follow-up. Patients who underwent cataract surgery presented a $2.8 \mathrm{mmHg}$ additional reduction in IOP with similar corneal endothelial cell counts as compared to patients who underwent LPI alone. Nonaka et al. [23] reported that cataract surgery subsequent to laser iridotomy was also effective for complete resolution of residual angle closure, with concomitant control of IOP. Therefore, LPI with subsequent cataract surgery is also a feasible strategy to manage chronic angle closure. However, one must keep in mind that, although the risk is low, laser iridotomy may pose a hazard to the corneal endothelium which is cumulative with the endothelial damage during subsequent phacoemulsification. Moreover, LPI also increases the risk of cataract, making a future cataract surgery more probable. Thus, phacoemulsification with posterior chamber IOL implantation is, in our opinion, becoming a first line therapy for eyes with PAC and cataract. Regarding the role of clear lens extraction in the management of PAC, only a few papers have addressed this matter [8,32]. The EAGLE study, which is due to report in 2014, will compare clinical outcomes and cost-effectiveness of clear lens extraction in PACG patients with standard care [33].

The authors acknowledge some limitations to this study. The limited sample size has led to some differences in the two study groups, which, however, did not compromise the main outcomes of this work. Nevertheless, and in order to try to compensate for this sample size limitation-and the appearance of what could have been false positive statistically significant results - we have decreased the $p$ value (from standard 0.05 to 0.01 ) needed for statistical significance. Second, the Pentacam is not a very precise exam to measure ACA, however, it gives precise and reproducible measures of ACV and depth [34]. Moreover, PAS evaluation by gonioscopy was made only qualitatively. Pre and postoperative assessment of PAS index, location, height, and width would have been important to better understand the long-term effect of the two different strategies on this important variable [35]. Finally, phacoemulsification in these eyes may sometimes be a complex procedure. In this study, they were performed by two experienced surgeons, therefore, the results may not be generalizable to the less experienced.

In conclusion, this study, which has the longest follow-up, proved that phacoemulsification with posterior chamber IOL implantation is a safe and more effective procedure than LPI in lowering IOP and preventing its long-term increase. This success is achieved by improving anterior chamber morphology in patients with chronic PAC and cataract.

Conflict of interest No conflicting relationship exists for any author.

\section{References}

1. Rosengren B (1953) The etiology of acute glaucoma. Am J Ophthalmol 36:488-492

2. Quigley HA, Broman AT (2006) The number of people with glaucoma worldwide in 2010 and 2020. Br J Ophthalmol 90(3):262-267

3. Lowe RF (1969) Causes of shallow anterior chamber in primary angle-closure glaucoma. Ultrasonic biometry of normal and angle-closure glaucoma eyes. Am J Ophthalmol 67(1):87-93

4. Snow JT (1977) Value of prophylactic peripheral iridectomy on the second eye in angle-closure glaucoma. Trans Ophthalmol Soc UK 97:189-191

5. Ang LPK, Aung T, Chew PTK (2000) Acute primary angle closure in an Asian population: long-term outcome of the fellow eye after prophylactic laser peripheral iridotomy. Ophthalmology 107:2092-2096

6. He M, Friedman DS, Ge J et al (2007) Laser peripheral iridotomy in primary angle-closure suspects: biometric and gonioscopic outcomes: the Liwan Eye Study. Ophthalmology 114:494-500

7. Gazzard G, Friedman DS, Devereux JG et al (2003) A prospective ultrasound biomicroscopy evaluation of changes in anterior segment morphology after laser iridotomy in Asian eyes. Ophthalmology 110:630-638

8. Roberts TV, Francis IC, Lertusumitkul S et al (2000) Primary phacoemulsification for uncontrolled angle-closure glaucoma. J Cataract Refract Surg 26(7):1012-1016

9. Lai JS, Tham CC, Chan JC (2006) The clinical outcomes of cataract extraction by phacoemulsification in eyes with primary angle-closure glaucoma (PACG) and co-existing cataract: a prospective case series. J Glaucoma 15(1):47-52

10. Lam DS, Leung DY, Tham CC et al (2008) Randomized trial of early phacoemulsification versus peripheral iridotomy to prevent intraocular pressure rise after acute primary angle closure. Ophthalmology 115(7):1134-1140

11. Husain R, Gazzard G, Aung T et al (2012) Initial management of acute primary angle closure: a randomized trial comparing phacoemulsification with laser peripheral iridotomy. Ophthalmology 119(11):2274-2281 
12. Foster PJ, Buhrmann R, Quigley HA, Johnson GJ (2002) The definition and classification of glaucoma in prevalence surveys. Br J Ophthalmol 86(2):238-242

13. Tarongoy P, Ho CL, Walton DS (2009) Angle-closure glaucoma: the role of the lens in the pathogenesis, prevention, and treatment. Surv Ophthalmol 54(2):211-225

14. Meyer MA, Savitt ML, Kopitas E (1997) The effect of phacoemulsification on aqueous outflow facility. Ophthalmology 104(8):1221-1227

15. Ho CL, Walton DS, Pasquale LR (2004) Lens extraction for angle-closure glaucoma. Int Ophthalmol Clin 44(1):213-228

16. Shams PN, Foster PJ (2012) Clinical outcomes after lens extraction for visually significant cataract in eyes with primary angle closure. J Glaucoma 21(8):545-550

17. Diestelhorst M, Krieglstein GK (1991) Influence of cataract and posterior chamber lens implantation on the dynamics of the aqueous humor. Prospective study in fluorophotometry. J Fr Ophtalmol 14(4):255-259

18. Wang N, Chintala SK, Fini ME, Schuman JS (2003) Ultrasound activates the TM ELAM-1/IL-1/NF-kappaB response: a potential mechanism for intraocular pressure reduction after phacoemulsification. Invest Ophthalmol Vis Sci 44(5):1977-1981

19. Liu CJ, Cheng CY, Ko YC, Lau LI (2006) Determinants of long-term intraocular pressure after phacoemulsification in primary angle-closure glaucoma. J Glaucoma 20(9):566-570

20. Liu CJ, Cheng CY, Wu CW et al (2006) Factors predicting intraocular pressure control after phacoemulsification in angleclosure glaucoma. Arch Ophthalmol 124(10):1390-1394

21. Huang G, Gonzalez E, Peng PH et al (2011) Anterior chamber depth, iridocorneal angle width, and intraocular pressure changes after phacoemulsification: narrow vs open iridocorneal angles. Arch Ophthalmol 129(10):1283-1290

22. Hayashi K, Hayashi H, Nakao F, Hayashi F (2000) Changes in anterior chamber angle width and depth after intraocular lens implantation in eyes with glaucoma. Ophthalmology 107(4):698-703

23. Nonaka A, Kondo T, Kikuchi M et al (2005) Cataract surgery for residual angle closure after peripheral laser iridotomy. Ophthalmology 112(6):974-979

24. Nonaka A, Kondo T, Kikuchi M et al (2010) Angle changes before and after cataract surgery assessed by Fourierdomain anterior segment optical coherence tomography. J Cataract Refract Surg 36(10):1758-1762
25. Kucumen RB, Yenerel NM, Gorgun E et al (2008) Anterior segment optical coherence tomography measurement of anterior chamber depth and angle changes after phacoemulsification and intraocular lens implantation. J Cataract Refract Surg 34(10):1694-1698

26. Nolan WP, See JL, Aung T et al (2008) Changes in angle configuration after phacoemulsification measured by anterior segment optical coherence tomography. J Glaucoma 17(6):455-459

27. Quigley HA (1981) Long-term follow-up of laser iridotomy. Ophthalmology 88:218-224

28. Kumar RS, Baskaran M, Chew PT et al (2008) Prevalence of plateau iris in primary angle closure suspects an ultrasound biomicroscopy study. Ophthalmology 115(3):430-434

29. Wang N, Wu H, Fan Z (2002) Primary angle closure glaucoma in Chinese and Western populations. Chin Med J (Engl) 115(11):1706-1715

30. Nonaka A, Kondo T, Kikuchi M et al (2006) Angle widening and alteration of ciliary process configuration after cataract surgery for primary angle closure. Ophthalmology 113(3):437-441

31. Hata H, Yamane S, Hata S, Shiota H (2008) Preliminary outcomes of primary phacoemulsification plus intraocular lens implantation for primary angle-closure glaucoma. J Med Invest 55(3-4):287-291

32. Gunning FP, Greve EL (1998) Lens extraction for uncontrolled angle-closure glaucoma: long-term follow-up. J Cataract Refract Surg 24(10):1347-1356

33. Azuara-Blanco A, Burr JM, Cochran C et al (2011) Effectiveness in Angle-closure Glaucoma of Lens Extraction (EAGLE) Study Group. The effectiveness of early lens extraction with intraocular lens implantation for the treatment of primary angle-closure glaucoma (EAGLE): study protocol for a randomized controlled trial. Trials 23(12):133

34. Shankar H, Taranath D, Santhirathelagan CT, Pesudovs K (2008) Anterior segment biometry with the Pentacam: comprehensive assessment of repeatability of automated measurements. J Cataract Refract Surg 34(1):103-113

35. Inoue T, Yamamoto T, Kitazawa Y (1993) Distribution and morphology of peripheral anterior synechiae in primary angle-closure glaucoma. J Glaucoma 2(3):171-176 